\title{
Campylobacter concisus as the etiologic agent of gastrointestinal diseases
}

\author{
Katarzyna Akutko ${ }^{A, D}$, Krzysztof Matusiewicz ${ }^{D, F}$ \\ $2^{\text {nd }}$ Department and Clinic of Pediatrics, Gastroenterology and Nutrition, Wroclaw Medical University, Poland \\ A - research concept and design; $B$ - collection and/or assembly of data; $C$ - data analysis and interpretation; \\ $D$ - writing the article; $E$ - critical revision of the article; $F$ - final approval of article
}

\section{Address for correspondence \\ Katarzyna Akutko \\ E-mail: katarzyna.akutko@gmail.com \\ Funding sources \\ none declared}

\section{Conflict of interest}

none declared

Received on March 06, 2015

Revised on May 28, 2015

Accepted on January 19, 2016

\begin{abstract}
A number of reports on the pathogenic influence of Campylobacter concisus on the human body and its role in many diseases of the gastrointestinal system, including gastroesophageal reflux disease, Barrett's esophagus and inflammatory bowel disease, have appeared lately. Campylobacter concisus is a Gram negative bacteria which requires an anaerobic environment or microaerophilic environment with hydrogen for growth and is therefore difficult to culture. Due to this difficulty, the rate of infections in epidemiological data are underestimated. There are reports that $C$. concisus was the only pathogen isolated from the stool of patients with acute diarrhea, which could indicate that it is an etiologic factor of acute gastrointestinal infections in humans. Moreover, the results of some studies suggest that infection with C. concisus is a factor predisposing to the development of gastroesophageal reflux disease and Barrett's esophagus, conditions which may be present before the development of cancer. There are also studies which indicate C. concisus infection as a trigger of inflammatory bowel disease, since it has been demonstrated that $C$. concisus is present more frequently in patients with newly diagnosed Crohn's disease than in a control group.
\end{abstract}

Key words: inflammatory bowel disease, Campylobacter concisus, reflux esophagitis, Barrett's esophagus

DOI

$10.17219 /$ acem/61428

\section{Copyright}

Copyright by Author(s)

This is an article distributed under the terms of the

Creative Commons Attribution Non-Commercial License

(http://creativecommons.org/licenses/by-nc-nd/4.0/) 
Infections with bacilli from the Campylobacter genus are the most frequent cause of acute bacterial diarrhea in developed countries. ${ }^{1}$ In European countries, the frequency of infections evoked by these bacteria outnumbers the frequency of infections caused by Salmonella. ${ }^{2}$ Campylobacter jejuni and Campylobacter coli are the genuses which cause disease symptoms most frequently. ${ }^{3}$ Progress in molecular techniques and the development of innovative culture methods has led to the isolation of other genuses than Campylobacter jejuni/coli such as C. concisus, C. upsaliensis and C. ureolyticus. ${ }^{4}$ The Campylobacter genus is comprised of 20 species and subspecies. $^{3}$ The clinical manifestations of Campylobacter infections are caused not only by gastrointestinal infection but also by periodontitis and inflammatory bowel disease. In some situations, a life-threatening generalized infection of the whole human body may be a complication of intestinal infection. ${ }^{4}$ C.concisus is a bacteria which is drawing the increasing interest of investigators who are involved in studies on gastrointestinal disease etiology.

Campylobacter concisus was first isolated in 1981 by Tanner et al. from material coming from patients with periodontitis and gingivitis. ${ }^{5-8}$ It is a Gram-negative rod which, in order to grow, requires anaerobic or microaerophilic conditions as well as the presence of hydrogen. ${ }^{5,7-9}$ The bacterial cells are curved or spiral, and their dimensions are $0.5-2 \times 2-6 \mu \mathrm{m} .^{5,7,8,10}$ They have the ability to move due to the presence of flagella. ${ }^{5,7,11} \mathrm{C}$. concisus is a species that requires specific environmental conditions in order to grow, namely a special substrate enriched with hydrogen and a microaerophilic environment, which are not used in the routine diagnostic procedures of gastrointestinal infections. For this reason, the pathogen is relatively rarely isolated, and its pathogenicity is underestimated. ${ }^{12,13}$ C.concisus is characterized by several virulence factors i.e. it produces hemolysin, cytolethal distendinglike toxin (CDT) - a cell cycle modulation toxin, RTX toxin and outer membrane fibronectin-binding protein 6). ${ }^{6,8,13-16}$ RTX toxin is synthesized by some Gram-negative bacteria. This toxin forms pores and has the ability of selective binding, which results in a more efficient connection with specific cells. ${ }^{6,16,17}$ The fibronectin-binding protein is probably responsible for the adherence of bacteria to host cells. ${ }^{6,16}$ There are also reports that $C$. concisus produces phospholipase and ligase, which are involved in the process of aggregation and adhesion to host cells, and Acr protein that can modulate the immune response of the host, and in this way perpetuate the state of inflammation. ${ }^{6,8}$ In vitro studies have demonstrated that $C$. concisus may cause damage to the epithelial cells and reduce the tightness of adhesion of cells to each other, which corresponds to the mechanism of the development of diarrhea. ${ }^{7,9,18}$ The impact of $C$. concisus on intestinal epithelial cells has not been definitively clarified. ${ }^{13}$

There are at least several different genotypes of $C$. concisus with different abilities to induce damage to the in- testinal epithelial cells, and with various virulence and clinical pictures of the infection. ${ }^{6,7,11,15,19,20}$ In in vitro studies, a $C$. concisus strain isolated from a child with newly-diagnosed Crohn's disease exhibited a greater ability to attach to and to invade the epithelial cells lining the intestine than strains isolated from patients with inflammation of the gastrointestinal tract. Furthermore, C. concisus strains isolated from healthy individuals were able to adhere to epithelial cells but lacked the ability to invade. ${ }^{13,20}$ The studies by Kaakoush et al. have confirmed that $C$. concisus' invasive potential is different in the case of strains isolated from patients with chronic inflammatory bowel disease than those isolated from healthy people or from patients with acute gastroenteritis. ${ }^{15}$ Based on the studies conducted, Man et al. have hypothesized that the presence of inflammation before $C$. concisus infection increases the ability of the pathogen invasion. ${ }^{13}$ Unlike other bacteria of the genus Campylobacter spp., C. concisus has not been detected in healthy animals. ${ }^{5,10}$ Moreover no non-human host for this microorganism has been discovered. ${ }^{5,9}$ No animals that could be a carrier for C. concisus have been discovered, although the bacterium has been isolated from pet dogs with diarrhea. ${ }^{18}$ However, the presence of the bacteria has been found in poultry meat and ground beef, which could suggest that C. concisus infection may be a zoonosis. This issue requires further investigation. ${ }^{10}$ The collected data suggests that man is a natural host for $C$. concisus, and the main location of colonization is the oral cavity. ${ }^{8} \mathrm{C}$. concisus has been recognized as an opportunistic organism for humans, which can cause periodontitis, gum infections and gastrointestinal infections. ${ }^{5,21,22}$ The epidemiology of infections caused by $C$. concisus is unknown, due to the lack of testing in the general population. There are speculations that the infection of further sections of the gastrointestinal tract is caused by endogenous (strains from the oral cavity) or exogenous (contaminated objects) mechanisms. ${ }^{8}$

\section{C. concisus and gingivitis and periodontitis}

The prevalence of $C$. concisus in the oral cavity is up to $97 \%$, although it is not the dominant bacteria in the oral microbiome. ${ }^{23}$ All the current tests indicate that C. concisus infection can cause periodontitis and gingivitis especially in the early stages accompanied by bleeding., 5, 12,20 Patients with periodontal disease show increased concentrations of anti-C. concisus antibodies in blood serum in comparison with a clinical control group. ${ }^{24}$ This bacterium has been more often isolated from the oral cavity of patients in the early stages of periodontal disease than in healthy patients or those with chronic inflammation. ${ }^{6}$ At this stage, there is not enough evidence to confirm that C. concisus is the pathogen responsible for the development of oral diseases. ${ }^{25}$ So far it has not been clarified 
whether $C$. concisus is a commensal in the oral cavity, an opportunistic microorganism that causes infection in certain circumstances or a pathogen responsible for the development of certain oral conditions. ${ }^{6}$

\section{C. concisus and esophagus diseases}

Gastroesophageal reflux disease and Barrett's esophagus are diseases resulting from the long-term impact of stomach acid or bile reflux on the esophageal mucosa. As a result of that, the squamous epithelium and lamina propria are damaged. ${ }^{26}$ Both gastroesophageal reflux disease and Barrett's esophagus are conditions that often precede the development of esophagus cancer. ${ }^{22,26,27}$ In the last 30 years the incidence of esophageal cancer has increased 6-fold. At the same time, an increase in the incidence of gastroesophageal reflux disease was observed. It seems that such a dynamic increase in the number of cases may have environmental background. However, the reason has not been fully clarified. ${ }^{28}$ There is also a hypothesis that the changes in the composition of the esophageal microflora contribute to the progression of the inflammatory process in gastroesophageal reflux disease to a pre-cancerous condition (Barrett's esophagus). ${ }^{27}$ In the esophagus, there is a biofilm which is changed in these conditions. The importance of this change in the etiology of esophageal pathology has not been elucidated. ${ }^{22}$

Studies on the composition of the distal esophagus microflora have shown that two types of this microflora can be distinguished. In the first type, more Gram-positive bacteria, mainly from the Streptococcus genus, are observed. ${ }^{22,26,28,29}$ Streptococci constitute nearly $79 \%$ of the microflora in the healthy esophagus. In type II, a dominance of Gram-negative bacteria is observed (Bacteroides, Proteobacteria, Fusobacterium and Spirochaetes). In this type, the role of bacteria of the Streptococcus genus is much smaller (30\%). The presence of bacteria of 24 other types can be observed, generally Gram-negative, including Campylobacter. ${ }^{22.26,28}$ Type II is often diagnosed with a pathology of this part of the gastrointestinal tract together with gastroesophageal reflux disease and Barrett's esophagus. ${ }^{26,28}$ It is estimated that gastroesophageal reflux disease and Barrett's esophagus are 15 times more likely to occur in patients with the type II microbiome. ${ }^{28}$ The percentage of Gram-negative bacteria is 3 times higher in the type II microbiome than in type I. Considering the fact that most of them show pro-inflammatory behavior in the oral cavity, it is likely that similar effects may show in other sections of the gastrointestinal tract. ${ }^{26}$

Lipopolysaccharide (LPS), which is the outermost component of the bacterial cell membrane of Gram-negative bacteria, acts like an endotoxin which stimulates many types of cells to the production of pro-inflammatory cytokines, activation of the transcription factor NF- $\mathrm{K}$ B (nu- clear factor $\kappa \mathrm{B}$ ), and may cause a decrease in the voltage of the lower esophageal sphincter and a delay in gastric emptying. ${ }^{26,27,30}$ In reflux disease and Barrett's esophagus we can observe increased activity of the NF- $\mathrm{K} B$ pathway, which is not present in a healthy esophagus. ${ }^{26,31} \mathrm{NF}-\mathrm{k} B$ is a major factor controlling the activity of pro-inflammatory factors in epithelial cells. ${ }^{32}$ Activation of the NF-K B path is equivalent to increased levels of proinflammatory cytokines like Il-1, IL-6, IL- 8 or TNF $\alpha$, contributes directly to stimulating an immune response and influences the rate of neoplastic transformation. ${ }^{26,31}$ Type II microbiome could be used as an indicator of the need for intervention in daily practice if it were proved that its presence contributes to the progression of erosive esophageal cancer. ${ }^{26}$ However, the conducted studies do not explain, whether the medical conditions of the esophagus arise from the qualitative change of microflora in the lower section of the esophagus from type I to II, or the change of type of microorganisms inhabiting this section of digestive tract is due to a disease state which is an inflammation of the esophagus. This requires further studies. ${ }^{29,30}$ However, if these changes are causally related to the progression of Barrett's esophagus, the removal of the dominant bacteria could be the primary goal of therapy through the use of antibiotics, probiotics and prebiotics. In the studies of Blackett et al., patients with esophageal reflux disease and Barrett's esophagus had a lower number of all types of bacteria compared to the control group, with the exception of the genus Campylobacter. $^{22}$ The most frequently isolated species was C. concisus. The presence of this bacteria was observed only in the diseased esophagus. This was not observed when comparing patients with esophageal cancer and the control group. Moreover, in patients with reflux esophagitis and Barrett's esophagus, together with diagnosed C. concisus, significantly higher levels of IL-18 than in the control group were observed. It has been reported that IL-18 affects the development of cancers, including gastric cancer. ${ }^{22}$ In the studies of Mafarlane et al., C. concisus was found in $57 \%$ of patients with Barrett's esophagus, but there were no patients with $C$. concisus in the control group. ${ }^{12}$ Zehng et al. demonstrated that Campylobacter infection is accompanied by the elevation of IL- 8 and TNF $\alpha$, which could also play an important role in the initiation of malignant transformation. ${ }^{32}$ It seems that changing environmental conditions in the esophagus, for example exposure to acidic gastric contents, favors the colonization of the gastrointestinal tract by $C$. concisus. The original change, however, has not as yet been established and requires further studies. ${ }^{22}$

\section{C. concisus and gastrointestinal tract infections}

The first reports that $C$. concisus may also colonize other than the oral cavity sections of the gastrointestinal tract come from 1987.5,6 Already in 1991, Lauwers et al., 
based on their study, drew attention to the relatively frequent isolation of $C$. concisus from stool samples in pediatric patients $(2.4 \%)$ and adults (1.5\%) with diarrhea. It is interesting that this symptom was present in the vast majority of patients in whom the bacterium was observed (72\% of children and nearly $90 \%$ of adults). ${ }^{6}$ On the basis of research conducted in later years in Europe, Australia and South Africa, it can be concluded that C. concisus may be the etiologic agent of gastrointestinal infections, especially in children aged $0-3$ years. ${ }^{5,6}$ Other studies confirm that C. concisus occurs in patients with diarrhea, but compared to healthy subjects, these values are not statistically significant. ${ }^{6}$ There are also reports that the only potentially pathogenic bacteria isolated from the stool samples of patients with diarrhea was C. concisus. ${ }^{19}$ So far, studies suggest that $C$. concisus may be an opportunistic pathogen, causing symptoms in predisposed patients. Hess et al. described the case of a 55-year-old man with symptoms of gastrointestinal infection, with C. concisus as an etiological factor. The authors draw attention to the diagnostic difficulties in detecting infection with this bacteria using traditional methods (culture). C. concisus incidence in patients with diarrhea has not been conclusively determined. ${ }^{21}$ In a study by Nielsen et al. among unselected patients treated for diarrhea, C. concisus infection was observed in a high percentage - $(35 / 100,000$ inhabitants), which was similar to the number of infections caused by $C$. jejuni/coli. C. concisus incidence in samples of feces of healthy individuals was significantly lower than in patients with gastrointestinal disease, which is indirect proof of the lack of favorable environmental conditions for the bowel colonization with C. concisus compared to the oral cavity. ${ }^{10}$ However, there are reports in the literature on the isolation of the same strain, C. concisus, from the mouth and from intestinal biopsies in patients with ulcerative colitis, which may indicate the possibility of colonization by the bacteria in other than the oral cavity, including parts of the intestinal tract. ${ }^{8}$

\section{C. concisus and inflammatory bowel disease}

The etiology of inflammatory bowel disease (IBD) has not been definitively elucidated so far. This is probably a result of the coexistence of a number of factors, including an infectious agent. ${ }^{23,33}$ Many studies have been dedicated to the impact of bacteria considered as commensals and bacteria with proven enteropathogenic activity on the formation or exacerbation of pre-developed chronic inflammatory bowel disease. ${ }^{33,34}$ In the literature there are reports that children with Crohn's disease have Campylobacter spp., especially C. concisus, infections more frequently than patients from the control group. ${ }^{5,23,33}$ In 2009, based on their studies, Zhang et al. demonstrated that, in pediatric patients with newly diagnosed Crohn's disease, Campy- lobacter spp. occurred much more frequently in an intestinal biopsy (82\%) compared to the control group (23\%). Examination of the biopsies using molecular methods has shown a significantly higher incidence of $C$. concisus DNA in children with newly-diagnosed Crohn's disease as compared to the control group (51 vs. $2 \%$ ). At the same time, the presence of C. concisus was confirmed using a biopsy culture method of the intestinal mucosa, which indicates the presence of viable bacterial cells in the intestine. ${ }^{23}$ In 2010, Man et al. obtained similar results - examining stool samples using PCR, C. concisus was found in $65 \%$ of pediatric patients with newly-diagnosed Crohn's disease as compared to $33 \%$ in the group of healthy individuals. ${ }^{11}$

Similar results were obtained in studies of adult patients with IBD. ${ }^{6}$ Mukhopadhya et al., among others, showed a significantly higher incidence of $C$. concisus in tissue biopsies of the colon in adult patients with ulcerative colitis compared to the control group..$^{35}$ Mahendran et al. obtained similar results by examining adult patients with Crohn's disease. ${ }^{6}$ It has also been shown that certain strains of $C$. concisus have the ability to colonize the gastrointestinal tract in mice, and can cause weight loss and the formation of liver micro-abscesses. ${ }^{11,13}$ In several studies carried out to date, the C. concisus infection rate in IBD patients has ranged from 33 to $67 \%$, compared to $2-37 \%$ in the control group. ${ }^{6}$ Furthermore, in the intestine of children with Crohn's diesease, other species of the genus Campylobacter spp. occurred, such as C. showae, C. rectus or C. gracilis, which were not found in patients from the control group. ${ }^{11}$ The results of these studies indicate that it may be necessary to conduct tests for the pathogenicity of different species of the genus Campylobacter spp. within the context of the etiology of IBD. 5,11

The authors of a study in Denmark have drawn attention to the need for planning and conducting research which would determine the risk of developing IBD in patients infected with C. concisus. ${ }^{10}$ The results of a study conducted by Ismail et al. suggest that patients with IBD are carriers of certain strains of C. concisus in the mouth. In some patients with IBD, this bacterium, through endogenous colonization, also inhabits the gut, where a natural process of recombination is taking place. ${ }^{8}$ Zhang et al., based on the analysis of literature, hypothesized that C. concisus can cause the development of IBD or cause exacerbation of the disease. This applies especially to the strains in the genome of which the zot (zonula occludens toxin) gene, originating from viruses, is incorporated. ${ }^{7}$ Because of this fact, bacteria strains can produce a Zot, similar to the toxin produced by $V$. cholerae, which destroys the close connection between the cells, causing an increase in intestinal barrier permeability. ${ }^{7,16}$ The oral cavity is a reservoir of bacteria from where the pathogen can migrate to the intestine and colonize it. Here the zot gene can be released, which can cause primary, long-term damage to the mucosal barrier and provide the ability to translocate microorganisms and their metabolic products 
from the lumen. In genetically predisposed individuals, this may be the cause of IBD development.

A similar mechanism may be responsible for the development of diarrhea, which explains the isolation of C. concisus from patients with diarrhea without IBD. It is known that there are certain risk factors for IBD, including environmental factors and a family history of inflammatory bowel disease. The authors suggest that one of these environmental factors could be C. concisus infection, which in their genome contains the zot gene, the product of which predisposes the intestinal mucosa to increased permeability. This may lead to the development of IBD. Further studies are necessary on the prevalence of $C$. concisus with the zot gene in patients with IBD. ${ }^{7}$ Kaakoush et al. isolated the Zot (zonula occludes toxin) from the C. concisus strain. There is speculation that the production of this toxin is dependent on the $C$. concisus genotype. ${ }^{6}$

\section{Conclusion}

Current studies indicated that $C$. concisus may be present in virtually every part of the gastrointestinal tract, and under particular conditions it may cause disease symptoms such as stomatitis and diarrhea, among others, and may also promote the development and persistence of inflammation in the intestine. Taking into consideration the reports cited in our work, a hypothesis may be put forth that the pathogenic potential of C.concisus has its roots in the presence of various virulence factors in each strain. There is a need for clarification of the role of particular strains of $C$. concisus, with various pathogenic potential, in the inciting and perpetuation of many diseases of the gastrointestinal tract with unknown etiology since, up to now, it has not been clarified if the bacteria is a commensal, opportunistic microbe or a pathogen for humans.

\section{References}

1. Silva J, Leite D, Fernandes M, Mena C, Gibbs PA, Teixeira P. Campylobacter spp. as a Foodborne Pathogen: A Review. Front Microbiol. 2011;2:200. doi:10.3389/fmicb.2011.00200.x.

2. Laroche M, Magras C. Gastrointestinal campylobacteriosis in industrialised countries: Comparison of the disease situation with salmonellosis, and microbiological contamination assessment. Rev Sci Tech. 2013;32(3):701-714.

3. Fernández H, Vera F, Villanueva MP, García A. Occurrence of Campylobacter species in healthy well-nourished and malnourished children. Braz J Microbiol. 2008;39(1):56-58.

4. Man SM. The clinical importance of emerging Campylobacter species. Nat Rev Gastroenterol Hepatol. 2011;8(12):669-685.

5. Istivan T, Ward P, Coloe P. Campylobacter concisus: An emerging pathogen of the gastrointestinal tract. Current Research, Technology and Education Topics in Applied Microbiology and Microbial Biotechnology. 2010; 626-634.

6. Kaakoush NO, Mitchell HM. Campylobacter concisus - A new player in intestinal disease. Front Cell Infect Microbiol. 2012;2:4. doi:10.3389/ fcimb.2012.00004.x.

7. Zhang L, Lee H, Grimm MC, Riordan SM, Day AS, Lemberg DA. Campylobacter concisus and inflammatory bowel disease. World J Gastroenterol. 2014;20(5):1259-1267.
8. Ismail Y, Mahendran V, Octavia S, et al. Investigation of the enteric pathogenic potential of oral Campylobacter concisus strains isolated from patients with inflammatory bowel disease. PLOS ONE. 2012;7(5):e38217. doi:10.1371/journal.pone.0038217.x.

9. Lastovica AJ. Emerging Campylobacter spp.: The tip of the iceberg. Clinical Microbiology Newsletter. 28(7):49-56.

10. Nielsen HL, Ejlertsen T, Engberg J, Nielsen H. High incidence of Campylobacter concisus in gastroenteritis in North Jutland, Denmark: A population-based study. Clin Microbiol Infect. 2013;19(5):445-450.

11. Man SM, Zhang L, Day AS, Leach ST, Lemberg DA, Mitchell H. Campylobacter concisus and other Campylobacter species in children with newly diagnosed Crohn's disease. Inflamm Bowel Dis. 2010;16(6):1008-1016.

12. Macfarlane S, Furrie E, Macfarlane GT, Dillon JF. Microbial colonization of the upper gastrointestinal tract in patients with Barrett's esophagus. Clin Infect Dis. 2007;45(1):29-38.

13. Man SM, Kaakoush NO, Leach ST, et al. Host attachment, invasion, and stimulation of proinflammatory cytokines by Campylobacter concisus and other non-Campylobacter jejuni Campylobacter species. J Infect Dis. 2010;202(12):1855-1865.

14. Istivan TS, Smith SC, Fry BN, Coloe PJ. Characterization of Campylobacter concisus hemolysins. FEMS Immunol Med Microbiol. 2008; 54(2):224-235.

15. Kaakoush NO, Deshpande NP, Wilkins MR, et al. The pathogenic potential of Campylobacter concisus strains associated with chronic intestinal diseases. PLoS ONE. 2011;6(12):e29045. doi:10.1371/journal.pone.0029045.

16. Kaakoush NO, Man SM, Lamb S, et al. The secretome of Campylobacter concisus. FEBS J. 2010;277(7):1606-1617. doi: 10.1111/j.17424658.2010.07587.x.

17. Stachowiak R, Bielecki J. Hemolizyny bakteryjne. Post Mikrobiol. 2000;39:253-270.

18. Nielsen HL, Nielsen $\mathrm{H}$, Ejlertsen T, et al. Oral and fecal Campylobacter concisus strains perturb barrier function by apoptosis induction in HT-29/B6 intestinal epithelial cells. PLOS ONE. 2011;6(8):e23858. doi:10.1371/journal.pone.0023858.x.

19. Aabenhus R, On SL, Siemer BL, Permin H, Andersen LP. Delineation of Campylobacter concisus genomospecies by amplified fragment length polymorphism analysis and correlation of results with clinical data. J Clin Microbiol. 2005;43(10):5091-5096.

20. Deshpande NP, Kaakoush NO, Mitchell H, et al. Sequencing and validation of the genome of a Campylobacter concisus reveals intraspecies diversity. PLOS ONE. 2011;6(7):e22170. doi:10.1371/journal. pone.0022170.x.

21. Hess DL, Pettersson AM, Rijnsburger MC, Herbrink $P$, van den Berg HP, Ang CW. Gastroenteritis caused by Campylobacter concisus. J Med Microbiol. 2012;61(5):746-749.

22. Blackett KL, Siddhi SS, Cleary S, et al. Oesophageal bacterial biofilm changes in gastro-oesophageal reflux disease, Barrett's and oesophageal carcinoma: Association or causality? Aliment Pharmacol Ther. 2013;37(11):1084-1092.

23. Zhang L, Man SM, Day AS, et al. Detection and isolation of Campylobacter species other than C. jejuni from children with Crohn's disease. J Clin Microbiol. 2009;47(2):453-455.

24. Taubman MA, Haffajee AD, Socransky SS, Smith DJ, Ebersole JL. Longitudinal monitoring of humoral antibody in subjects with destructive periodontal diseases. J Periodontal Res. 1992;27(5):511-521.

25. Henne K, Fuchs F, Kruth S, Horz HP, Conrads G. Shifts in Campylobacter species abundance may reflect general microbial community shifts in periodontitis progression. J Oral Microbiol. 2014;6. doi:10.3402/jom.v6.25874.x.

26. Yang L, Francois F, Pei Z. Molecular pathways: Pathogenesis and clinical implications of microbiome alteration in esophagitis and Barrett esophagus. Clin Cancer Res. 2012;18(8):2138-2144.

27. Pei Z, Yang L, Peek RM, Jr Levine SM, Pride DT, Blaser MJ. Bacterial biota in reflux esophagitis and Barrett's esophagus. World $J$ Gastroenterol. 2005;11(46):7277-7283.

28. Yang L, Oberdorf WE, Gerz E, et al. Foregut microbiome in development of esophageal adenocarcinoma. Nature Precedings. 2010. doi:10.1038/npre.2010.5026.1.x.

29. Suerbaum S. Microbiome analysis in the esophagus. Gastroenterology. 2009;137(2):419-421. 
30. Tack J, Carethers JM. Different microbiome patterns in normal, inflammed, and Berrett's esophagus. Gastroenterology. 2009;137(2):398-399.

31. Peng DF, Hu TL, Soutto M, Belkhiri A, El-Rifai W. Glutathione peroxidase 7 suppresses bile salt-induced expression of pro-inflammatory cytokines in Barrett's carcinogenesis. J Cancer. 2014;5(7):510-517.

32. Zheng J, Meng J, Zhao S, Singh R, Song W. Campylobacter-induced interleukin-8 secretion in polarized human intestinal epithelial cells requires Campylobacter-secreted cytolethal distending toxinand Toll-like receptor-mediated activation of NF-kappaB. Infect Immun. 2008;76(10):4498-4508.
33. Gradel KO, Nielsen HL, Schonheyder HC, Ejlertsen T, Kristensen B, Nielsen $\mathrm{H}$. Increased short- and long-term risk of inflammatory bowel disease after salmonella or campylobacter gastroenteritis. Gastroenterology. 2009;137(2):495-501.

34. Shim JO. Gut microbiota in inflammatory bowel disease. Pediatr Gastroenterol Hepatol Nutr. 2013;16(1):17-21.

35. Mukhopadhya I, Thomson JM, Hansen R, Berry SH, El-Omar EM, Hold GL. Detection of Campylobacter concisus and other Campylobacter species in colonic biopsies from adults with ulcerative colitis. PLoS ONE. 2011;6(6):e21490. doi:10.1371/journal.pone.0021490. 